\title{
A group cognitive therapy programme reduced depressive episodes in at risk adolescents at 12 months of follow up
}

Clarke GN, Hornbrook M, Lynch F, et al. A randomized trial of a group cognitive intervention for preventing depression in adolescent offspring of depressed parents. Arch Gen Psychiatry 2001 Dec;58:1127-34.

\section{QUESTION: In adolescents with depressive symptoms and who have depressed parents, does a group cognitive intervention prevent or delay the onset of the subsequent development of a full depressive episode?}

Source of funding:

National Institute of

Mental Health.

For correspondence:

Dr G N Clarke, Kaiser

Permanente Center for

Health Research

Portland, Oregon, USA

greg.clarke@kp.org

\section{Design}

Randomised \{allocation concealed*\}†, blinded \{data collectors, data analysts, and study investigators $\}^{*}$, controlled trial with 24 months of follow up (median 14 months of follow up).

\section{Setting}

Health maintenance organization (HMO) in Portland, Oregon, USA.

Cognitive therapy $v$ usual care in adolescents at risk of depression at 12 months:

\begin{tabular}{lclll} 
Outcome & $\begin{array}{l}\text { Cognitive } \\
\text { therapy }\end{array}$ & $\begin{array}{l}\text { Usual } \\
\text { care }\end{array}$ & RRR (95\% Cl) & NNT (Cl) \\
$\begin{array}{l}\text { Risk of incident major } \\
\text { depressive episodes }\end{array}$ & $9 \%$ & $29 \%$ & $80 \%(32$ to 94$)$ & 4 (4 to 11) \\
\hline
\end{tabular}

$\neq$ Abbreviations defined in glossary; RRR, NNT, and $\mathrm{Cl}$ calculated from data in article.

\section{COMMENTARY}

This study by Clarke et al shows that a group cognitive intervention had medium effects in preventing major depression and reducing depressive symptoms in adolescent children of depressed parents. These effects were strongest at 12 months of follow up, but were reduced by 24 months. This work represents an important first step toward the development of a targeted preventive intervention for this population.

The findings of this study may not be generalisable due to the large initial attrition in the sample; $85 \%$ did not participate. One weakness of targeted and universal interventions is that those who most need the intervention are often the least likely to take it up. If we are to understand this intervention's potential public health effect, it must be further evaluated to assess whether those who most need it, in fact participate. A second related issue is that even with higher participation rates, the recruitment methods could not be mounted generally.

Finally, there are the issues of loss of effect over 2 years of follow up and the failure to find an effect on comorbid disorders and adaptive functioning. Attenuation of treatment effects over time is usual for most interventions. The authors suggest that booster sessions may be used to extend protective effects. Prior research shows that children of depressed parents are at risk, not only for the development of depressive disorders, but also for a range of other disorders and functional impairments. The cognitive intervention is targeted at depressogenic cognitions and as expected its chief effect is on depression outcomes. However, in the longer term, comorbid disorders and functional impairments may be equally important in determining adult outcomes. It is an open question whether a multifaceted intervention model that also targets these disorders and impairments would be equally or more effective in this population. Certainly, both research and clinical experience point to the different treatment needs of individual children and families and it remains possible that more accurate targeting according to treatment need and preference will be the most useful approach. If so, a range of effective interventions will be required, and it is a safe bet that the cognitive group developed by the authors will be among them.

Mark Sanford, MBChB , FRCPC University of Toronto Toronto, Ontario, Canada

\section{Patients}

94 adolescents (mean age $15 \mathrm{y}, 60 \%$ girls) with both subsyndromal depression and a parent with either a current episode of major depression or dysthymia, or an episode in the previous 12 months. $83 \%$ were followed for up to 24 months.

\section{Intervention}

45 adolescents were allocated to usual HMO care plus a 15 session group cognitive therapy prevention programme and 49 were allocated to usual HMO care only. In the group cognitive therapy prevention programme adolescents were taught cognitive restructuring techniques. Each session lasted 1 hour.

\section{Main outcome measures}

Scores on the Achenbach Child Behavior Checklist (CBCL), the Center for Epidemiological StudiesDepression Scale (CES-D), the Hamilton Depression Rating Scale (HAM-D), the Schedule for Affective Disorder and Schizophrenia for School-Age Children, Epidemiological Version (K-SADS-E), and the Global Assessment of Functioning Scale (GAF); and risk of incident major depressive episodes.

\section{Main results}

Treatment programme by time effects favouring the cognitive therapy group were shown for the CES-D $(\mathrm{p}=0.005)$, the HAM-D $(\mathrm{p}=0.05)$, the K-SADS-E $(\mathrm{p}=0.04)$, and the GAF $(\mathrm{p}=0.04)$ but not the CBCL $(\mathrm{p}=0.62)$. The statistically significant group differences were evident from baseline to after treatment and after treatment to 1 year follow up, but not to 2 years follow up. After adjustment for sex, age, CES-D score, and depression history, the cumulative risk of incident major depressive episodes at 12 months follow up was lower in the cognitive therapy group than in the usual care group (table). This difference was no longer statistically significant $(\mathrm{p}=0.07)$ at 24 months.

\section{Conclusion}

In adolescent offspring of depressed parents, a group cognitive therapy programme reduced depressive symptoms and the cumulative risk of depressive episodes at 12 months follow up.

*See glossary.

$\dagger$ Information provided by author. 